Supporting Information

\title{
6ק-N-Heterocyclic Substituted Naltrexamine Derivative BNAP: A Peripherally Selective Mixed MOR/KOR Ligand
}

Dwight A. Williamsa, ${ }^{\mathrm{a} b}$, Yi Zheng ${ }^{\mathrm{a}}$, Bethany G. David ${ }^{\mathrm{b}}$, Yunyun Yuan ${ }^{\mathrm{a}}$, Saheem A. Zaidia ${ }^{\mathrm{a}}$, David L. Stevens ${ }^{\mathrm{b}}$, Krista L. Scoggins ${ }^{\mathrm{b}}$, Dana E. Selley ${ }^{\mathrm{b}}$, William L. Dewey ${ }^{\mathrm{b}}$, Hamid I. Akbarali $^{\mathrm{b}}$, Yan Zhang ${ }^{\mathrm{a}^{*}}$

${ }^{a}$ Department of Medicinal Chemistry, Virginia Commonwealth University, 800 E. Leigh Street, Richmond, Virginia 23298, United States

${ }^{\mathrm{b}}$ Department of Pharmacology and Toxicology, Virginia Commonwealth University, 410

North $12^{\text {th }}$ Street, Richmond, Virginia 23298, United States 
BNAP characterization (including original spectra):

${ }^{1} \mathrm{H}$ NMR (400 MHz, DMSO-d $\left.\mathrm{d}_{6}\right): \delta, 9.39-9.42(1 \mathrm{H}, \mathrm{d}, \mathrm{J}=8.12 \mathrm{~Hz}), 9.38-9.36$ (2H, d, J = 6.84 Hz), $9.01(1 \mathrm{H}, \mathrm{s}),, 8.50-8.48$ (2H, d, J = 6.8 Hz), 7.48-7.25 (5H, m), $6.61-6.59(1 \mathrm{H}, \mathrm{d} \mathrm{J}=8.0 \mathrm{~Hz}), 6.56-6.54(1 \mathrm{H}, \mathrm{d}, \mathrm{J}=8.1 \mathrm{~Hz}), 5.92$ $(1 \mathrm{H}, \mathrm{s}), 4.91(1 \mathrm{H}, \mathrm{s}), 4.65$ - $4.64(1 \mathrm{H}, \mathrm{d}, \mathrm{J}=7.7), 3.74-3.66(1 \mathrm{H}, \mathrm{m}), 3.04-$ $2.96(3 \mathrm{H}, \mathrm{m}) 2.68$ - $2.51(2 \mathrm{H}, \mathrm{m}), 2.39-2.30(2 \mathrm{H}, \mathrm{m}), 2.24-2.14(1 \mathrm{H}, \mathrm{m})$, 2.02 - 1.84 (2H, m) 1.63 - 1.26 (4H, m), 0.89 - 0.80 (1H, m), $0.51-0.43$ $(2 \mathrm{H}, \mathrm{m}) 0.14-0.11(2 \mathrm{H}, \mathrm{m})$.

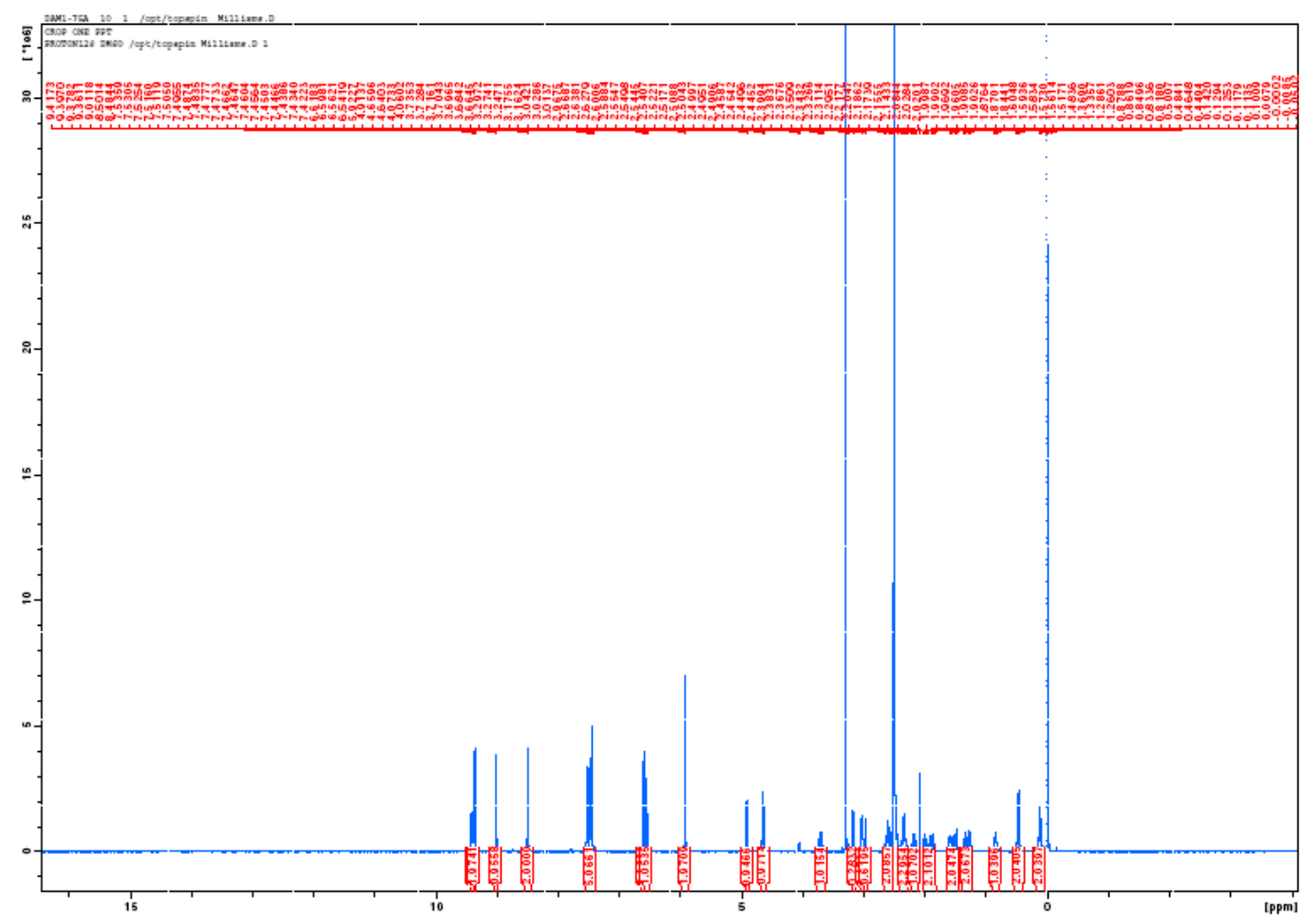


${ }^{13} \mathrm{C}$ NMR (100 MHz, DMSO-d 6 ) $\delta 161.1,148.0,145.8,141.8,141.1,133.8$, 129.4, 129.2, 128.7, 126.0, 120.6, 119.5, 117.8, 89.3, 69.5, 63.5, 61.6, 56.6, 51.8, 46.3, 45.5, 29.2, 27.1, 23.2, 22.9, 5.6, 5.0, 2.5.

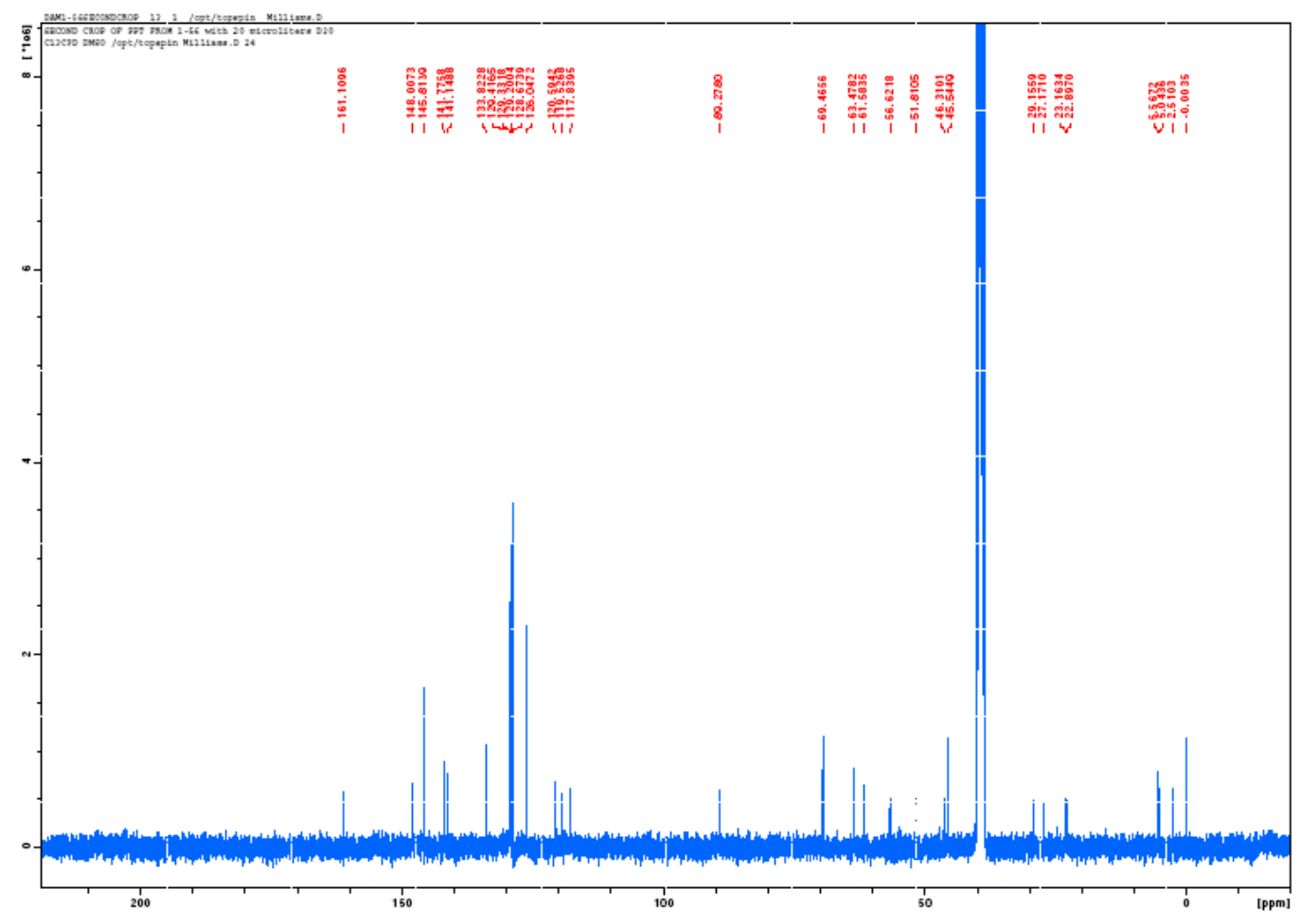


IR: 3433.29, 2251.06, 2125.05, 1661.09, 1052.38, 1023.83, 1004.68, 821.38, 758.75 .

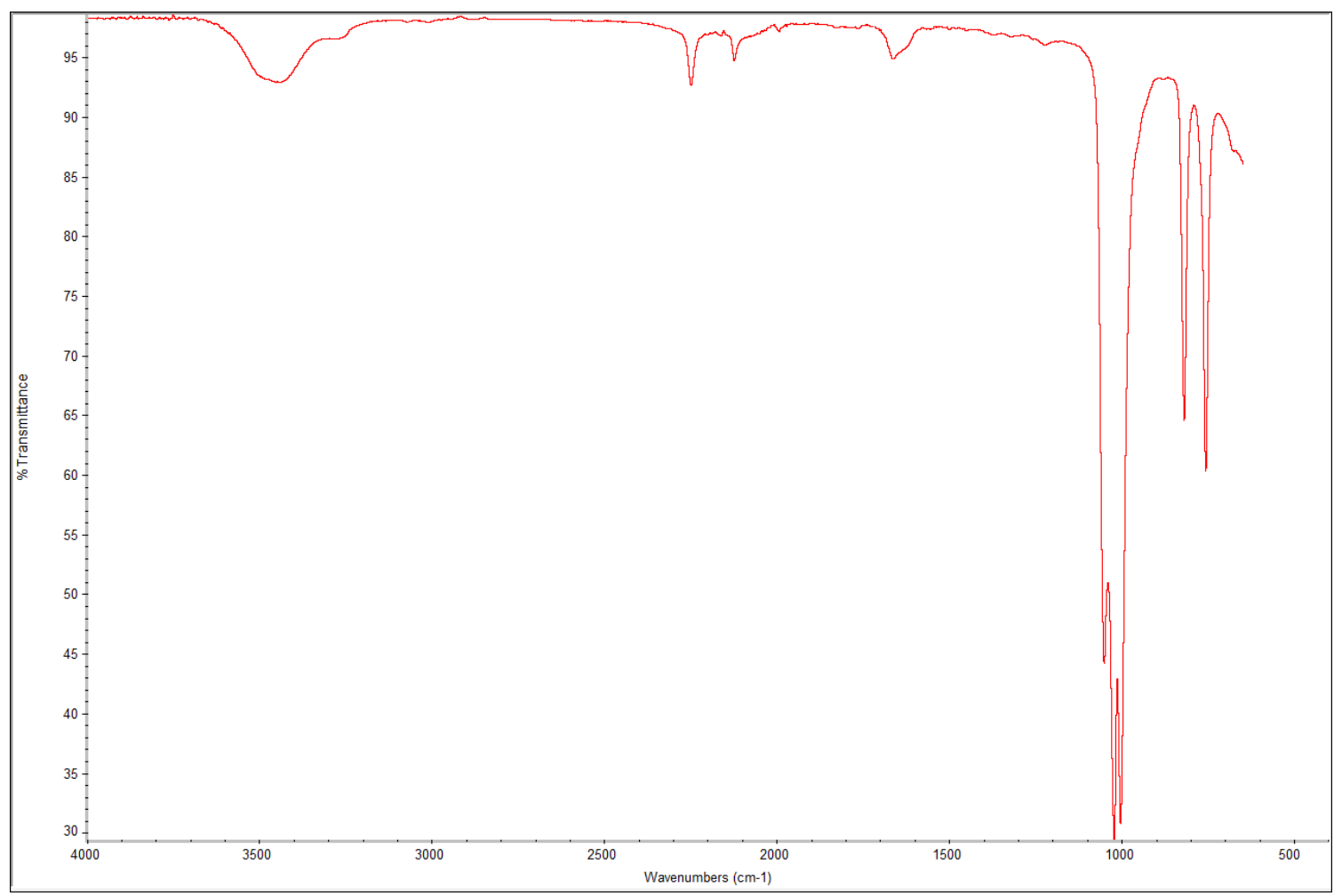




\section{HPLC:}
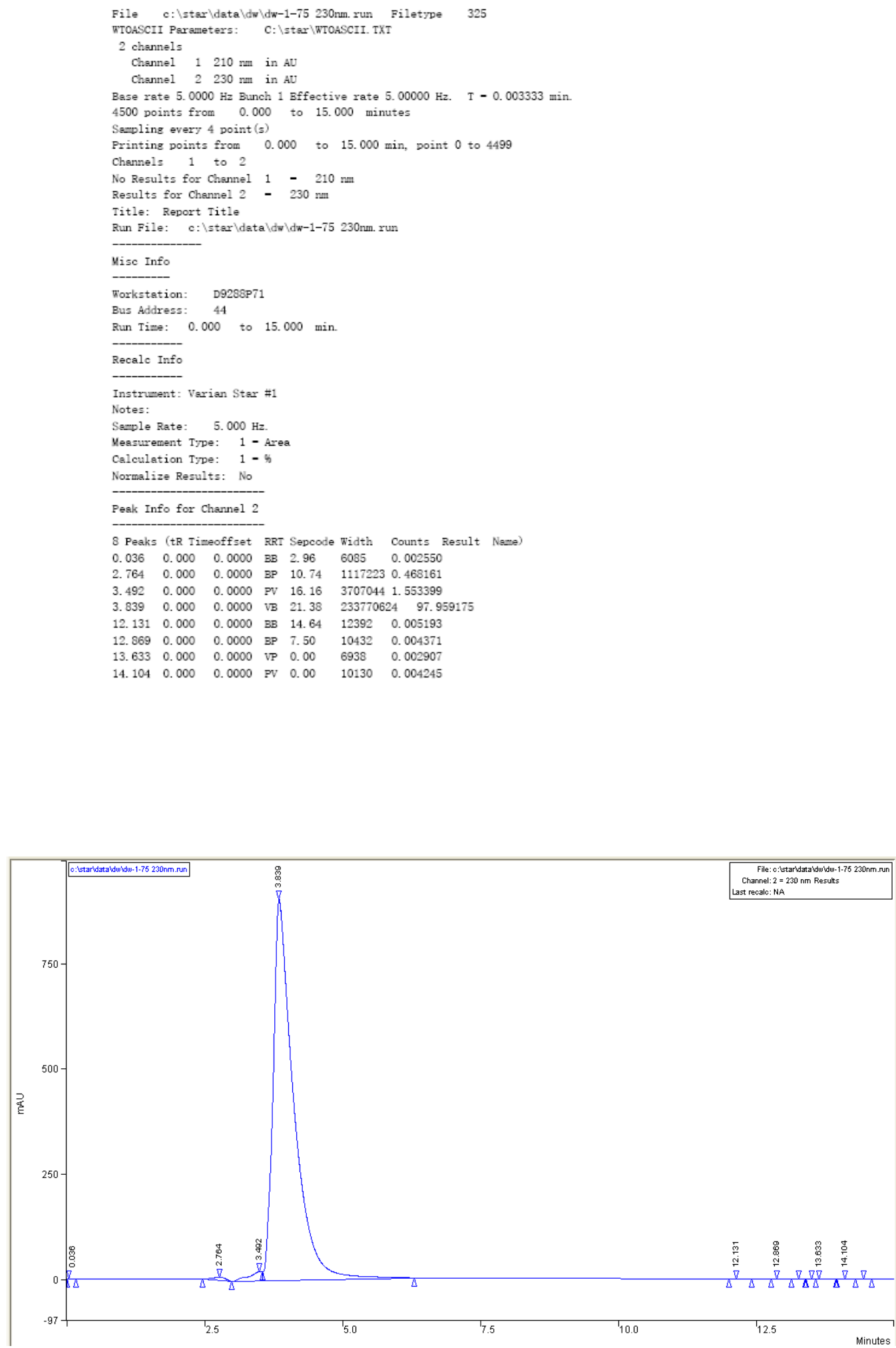


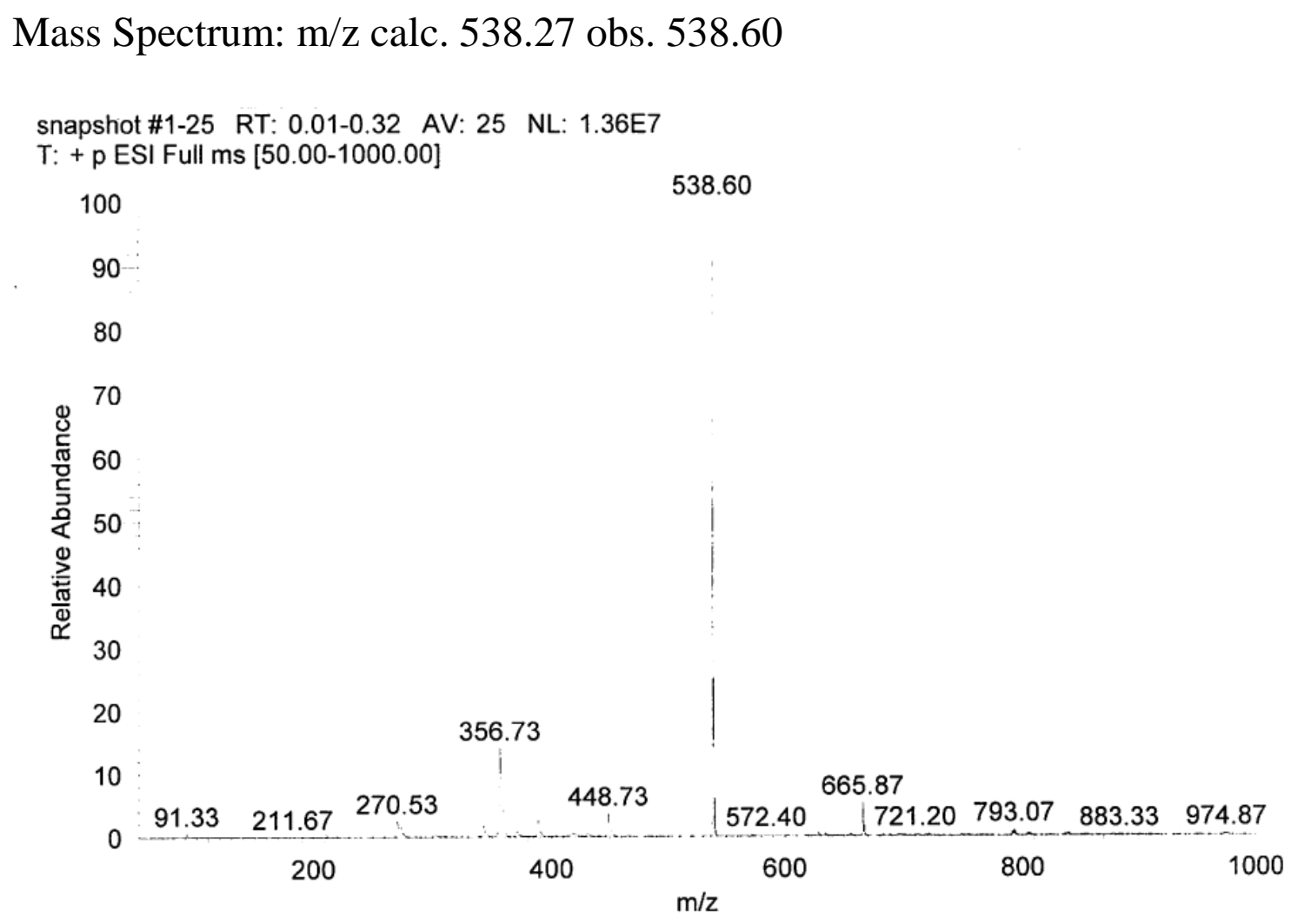

